Draft Version November 25, 2018

Preprint typeset using $\mathrm{LAT}_{\mathrm{E}} \mathrm{X}$ style emulateapj v. 11/10/09

\title{
REES-SCIAMA EFFECT AND IMPACT OF FOREGROUND STRUCTURES ON GALAXY REDSHIFTS
}

\author{
Hu ZHAN \\ Key Laboratory of Optical Astronomy, National Astronomical Observatories, Chinese Academy of Sciences, Beijing 100012, China; \\ zhanhu@nao.cas.cn \\ Draft version November 25, 2018
}

\begin{abstract}
We estimate the Rees-Sciama (RS) effect of super structures on the cosmic microwave background (CMB) temperature fluctuations and identify a related effect on galaxy redshifts. By numerically solving the geodesic equation, we find that both superclusters and supervoids can decrease the temperature of the CMB by several micro Kelvin in the central region and increase the temperature slightly in the surrounding area due to the RS effect. The two components of the RS effect, redshift and gravitational time delay, largely cancel each other, leaving an equivalent but much smaller effect on the CMB photons that started out at the same time from the distorted last scattering surface. For galaxies, the time delay effect is separable from the redshift effect, and the slight change to the redshift induced by super structures can be at the percent level of large-scale rms bulk velocities, which might only be detected statistically. On much smaller scales, a tiny redshift difference between two images of a strongly lensed source should exist in general, which is related to the Hubble expansion rate at the source redshift. However, as Loeb (1998) pointed out, observational issues and the proper motion of the structure would make such a measurement impossible.
\end{abstract}

Subject headings: cosmic microwave background - cosmology: theory - galaxies: distance and redshifts — large-scale structure of universe - relativity

\section{INTRODUCTION}

Measurements of the cosmic microwave background (CMB) with the Wilkinson Microwave Anisotropy Probe (WMAP, Bennett et al. 2003) have played a crucial role in establishing the concordance cosmological model, $\Lambda$ CDM - a flat cold-dark-matter universe with roughly three quarters of its content in the form of dark energy (e.g., Spergel et al. 2003). Besides the primary anisotropies, one also expects secondary CMB anisotropies arising from late-time gravitational effects, such as the integrated Sachs-Wolfe (ISW) effect (Sachs \& Wolfe 1967), and scattering processes, such as the thermal Sunyaev-Zel'dovich effect (Sunyaev \& Zeldovich 1972). Detections of these effects (e.g., Birkinshaw, Hughes, \& Arnaud 1991; Carlstrom, Joy, \& Grego 1996; Fosalba, Gaztañaga, \& Castander 2003; Boughn \& Crittenden 2004; Afshordi, Loh, \& Strauss 2004; Padmanabhan et al. 2005; Giannantonio et al. 2008; Granett, Neyrinck, \& Szapudi 2009) are useful confirmations of the $\Lambda$ CDM model.

The ISW effect on CMB photons is caused by the time variation of gravitational potentials as the photons travel through them. In order to have a measurable ISW effect, the photon travel time must be an appreciable fraction of the time scale, over which the potentials vary significantly. On large scales, overdensities in an Einstein-de Sitter (EdS) universe grows at the same rate as the cosmic expansion, so that the linear ISW effect vanishes. However, in a $\Lambda \mathrm{CDM}$ universe, the cosmic expansion rate exceeds the linear growth rate, causing a decay of potentials. A photon traveling through a decaying potential well (wall) gains (loses) energy. Therefore, detection of the linear ISW effect provides a piece of evidence for dark energy in a flat universe.
Nonlinear clustering causes extra evolution of the potentials against the background expansion, so that the ISW effect is present even in an EdS universe. This effect is also known as the Rees-Sciama (RS) effect (Rees \& Sciama 1968). On large scales, the nonlinear contribution (or the intrinsic RS effect) to the full ISW effect is expected to be subdominant to the linear ISW effect in a cosmological constant (or dark energy) dominated universe (Seljak 1996; Tuluie, Laguna, \& Anninos 1996).

In a recent paper, Granett, Neyrinck, \& Szapudi (2008) report a mean CMB temperature change of $|\Delta T|=9.6 \pm 2.2 \mu \mathrm{K}$ caused by superclusters and supervoids at $z \sim 0.5$ (hereafter, we drop the prefix "super" where there is no ambiguity). These structures have radii of $\sim 100 h^{-1} \mathrm{Mpc}$ or $4^{\circ}$ at $z \sim 0.5$. The scales involved are large enough, so that the linear ISW effect should be the major source of the temperature change in the $\Lambda \mathrm{CDM}$ model. However, the result is somewhat puzzling, because the temperature change is twice of what is estimated from simulations (Granett et al. 2008). Furthermore, there is no significant difference between the mean temperature of the clusters and that of voids in the reconstructed ISW map (Granett, Neyrinck, \& Szapudi 2009).

Given the studies above, it is of interest to reexamine the different mechanisms, through which cosmic structures alter the CMB temperature. We focus on the intrinsic RS effect in this paper. Our goal is not to reconcile the reported mean CMB temperature change with theory, but we note that the thermal SunyaevZel'dovich effect and the proper motion effect of structures (Birkinshaw \& Gull 1983; Gurvits \& Mitrofanov 1986; Stebbins 1988) are not likely to explain the discrepancy. In WMAP bands, unresolved thermal SunyaevZel'dovich signals would reduce the CMB temperature 
of clusters with respect to that of voids and hence suppress $|\Delta T| / T$, whereas the proper motion of a cluster or void would produce a dipole pattern without affecting the mean temperature.

We adopt the EdS universe as the background cosmology to isolate the intrinsic RS effect (we drop the word "intrinsic" hereafter). Because the growth rate of structures varies with the assumed cosmology, the RS effect is model dependent. For example, the combined RS and proper motion effects decrease by a factor of $\sim 2$ from the EdS universe to an open universe with matter fraction 0.3 (Tuluie et al. 1996).

One approach to investigate the RS effect is to analyze its power spectrum (e.g., Seljak 1996). The linear ISW effect increases the CMB temperature fluctuation and hence the power of the modes by raising the temperature of overdense regions and lowering that of underdense regions. Because the RS effect produces a net temperature decrement for both clusters and voids (see Section 5 for an explanation), it would not increase the temperature fluctuation as much even if it had the same amplitude as the linear ISW effect. Thus, power spectrum analyses may underestimate the importance of the RS effect.

Ray-tracing through $N$-body simulations can provide a realistic estimate of the full ISW effect (e.g., Tuluie et al. 1996; Maturi et al. 2007; Cai et al. 2009, 2010; Smith et al. 2009) To do so exactly, one needs to solve for the metric and its spatial-temporal derivatives, which appear in the geodesic equation. The ISW effect is roughly $|\Delta T| / T=|\Delta z| /(1+z) \sim 10^{-6}-10^{-5}$ for super structures, so that a redshift error of the order $10^{-7}$ or larger accumulated along the photon geodesic, or a comparable error from approximating the photon geodesic with a straight coordinate ray, can be a significant contamination to the results. Therefore, it is computationally challenging to trace photon geodesics in $N$-body simulations.

In this paper, we study the effect of individual structures with toy models (see, e.g., Thompson \& Vishniac 1987; Martinez-Gonzalez \& Sanz 1990). The advantage of this approach is that (1) for certain class of models, one can obtain analytic solutions of the underlying metric without worrying about spatial, temporal, or mass resolutions and (2) in models with symmetry, certain null geodesics can be computed in different ways, so that one can examine the precision of the calculations. Specifically, we model the clusters and voids with the spherically symmetric LemaitreTolman-Bondi (LTB, Lemaître 1933; Tolman 1934; Bondi 1947, referenced herein) solution and solve generic photon geodesics through these structures (see also Panek 1992; Alnes \& Amarzguioui 2006; Marra et al. 2007; Valkenburg 2009).

Separately, the RS effect has been studied for compensated voids and clusters (Inoue \& Silk 2006; Tomita \& Inoue 2008; Sakai \& Inoue 2008) and for compensated shells (Afshordi, Slosar, \& Wang 2011) in the $\Lambda \mathrm{CDM}$ universe with thin-shell approximation, perturbative calculations, and numerical calculations. The focus of this paper is on the effect caused solely by the evolution of the structures, so we do not include the cosmological constant $\Lambda$ except for a simple case in Section 6 . Our results are in qualitative agreement with those in the aforementioned works, and we extend the study to uncompensated structures as well.

As Rees \& Sciama (1968) pointed out, redshift and time delay are the two major components of the RS effect. The redshift component includes both compensation for the time delay component and the evolution of the potential. Since the effect of the potential evolution is usually subdominant, the two components of the RS effect often shift the CMB temperature with comparable magnitudes but in opposite directions.

Galaxy redshifts are directly measurable, so fractional changes to galaxy redshifts due to intervening structures are larger than that to the CMB temperature. This foreground-induced change of galaxy redshifts is closely related to but different from the RS effect or the ISW effect in general because of the separation of the redshift effect and the time delay effect on galaxies. However, unlike the CMB, which has a standard temperature, galaxies are spread over redshift space. Detecting such a redshift change is far more difficult than that of the RS effect.

Images of a strongly lensed source should have slightly different redshifts in general. Despite the extreme difficulty in measuring such redshift differences (Loeb 1998), we find in the ideal case that the redshift difference between two images of the source is considerably larger than that between two epochs of observations of the same image separated by the amount of the time delay between the two images. The latter is known as the Sandage-Loeb test (Sandage 1962; Loeb 1998).

The rest of the paper is organized as follows. Section 2 provides a brief introduction to the LTB solution and describes models of clusters and voids. Section 3 gives the details of solving the null geodesics numerically. Results of the RS effect on CMB temperature profiles and tiny perturbations to galaxy redshifts caused by intervening structures are presented in Sections 4 and 5, respectively. Further discussion is made in Section 6.

\section{LTB MODELS OF SUPER STRUCTURES}

We model the structures as spherically symmetric, dust-filled objects embedded in the EdS universe. The line element in such models is described by the LTB metric

$$
d s^{2}=-d t^{2}+\frac{R^{\prime 2}(t, r) d r^{2}}{1-K(r) r^{2}}+R^{2}(t, r) d \Omega^{2},
$$

where $R(t, r)$ is the angular diameter distance of the coordinate $r$ as viewed from the center, $K(r)$ is the curvature function, a prime denotes a partial derivative with respect to $r$, and the speed of light has been set to unity. The evolution of $R(t, r)$ is determined by $K(r)$ and the mass function $M(r)$

$$
\dot{R}^{2}(t, r)=\frac{2 G M(r)}{R(t, r)}-K(r) r^{2},
$$

where $G$ is Newton's constant, and an overdot stands for a partial derivative with respect to $t$ (hereafter, we suppress the variables $t$ and $r$ if there is no ambiguity in the context). The mass function is related to the acceleration

$$
\ddot{R}=-\frac{G M}{R^{2}},
$$


and the coordinate density is given by

$$
\rho=\frac{M^{\prime}}{4 \pi R^{2} R^{\prime}} .
$$

The general parametric solution of Equation (2) for $K>0$ is

$$
\begin{aligned}
R & =\frac{G M}{K r^{2}}(1-\cos u) \\
t-t_{B}(r) & =\frac{G M}{K^{3 / 2} r^{3}}(u-\sin u) .
\end{aligned}
$$

The bigbang time, $t_{B}(r)$, defines a coordinate surface, $R\left[t_{B}(r), r\right]=0$, at bigbang and is determined by $M$ and $K$ up to a scaling of $r$. It ensures that every part of the inhomogeneous universe contracts to a singular point at the same coordinate time as we look back. The solution for $K<0$ is obtained by replacing $u$ with $i u$. In the special case, $K=0$, we have

$$
R=\left(\frac{9 G M}{2}\right)^{1 / 3}\left[t-t_{B}(r)\right]^{2 / 3} .
$$

If both $K$ and $M$ are constant, i.e., the universe is homogeneous and isotropic, the solution has the form $R=a r$ with $a$ being the scale factor of the cosmic expansion. More specifically, we reserve $a$ for the scale factor of the EdS universe, i.e., the background, in this paper.

A particular realization of the LTB model is generated by specifying $K$ (or $M$ ) and initial values of $R$ and $\dot{R}$. Following Marra et al. (2007) and Paranjape \& Singh (2008), we set

$$
R\left(t_{i}, r\right)=a_{i} r \quad \text { and } \quad \dot{R}\left(t_{i}, r\right)=a_{i} H_{i} r
$$

where $a_{i} \equiv a\left(t_{i}\right)$ and $H_{i} \equiv H\left(t_{i}\right)$ are, respectively, the scale factor and Hubble expansion rate of the EdS background at the initial time $t_{i}$. For convenience, we choose $a_{i}=10^{-3}$ and $H_{i} a_{i}^{3 / 2}=H_{0}=50 \mathrm{~km} \mathrm{~s}^{-1} \mathrm{Mpc}^{-1}$. This rather small value of the Hubble constant is often adopted in the EdS universe to satisfy the age constraint, and, for this work, it also roughly matches the angular size of the model structures in the EdS universe with that in Granett et al. 2008 for the same linear size. Because we set the linear size of the structures in units of Mpc, a lower $H_{0}$ would make these structures smaller relative to the observable universe and reduce their RS signal. Results with a larger $H_{0}$ are given in Section 4 for comparison. Equation (8) ensures that the overdense (underdense) region always contracts (expands) against the background after $t_{i}$ (but before the big crunch, if applicable) and thus becomes more and more overdense (underdense). One may also set up voids with the overdensity in underdense region not always decreasing with time (e.g., Alnes, Amarzguioui, \& Grøn 2006; Kolb, Marra, \& Matarrese 2008).

We study both compensated and uncompensated LTB models. The former requires the mean density of the region to match that of the EdS background beyond a finite radius, and hence there is no effect on the rest of the universe due to Birkhoff's theorem. We design our toy models to have a smooth density profile (i.e., at least continuous in its first derivative with respect to $r$ ) with a uniform inner region (region I) inside a radius $r_{\mathrm{I}}$, so that numerical results there can be checked against analytic solutions. The compensated model is realized by setting $K=0$ beyond an outer radius, $r_{\mathrm{II}}$. We refer to the region between $r_{\mathrm{I}}$ and $r_{\mathrm{II}}$ as region II and that outside $r_{\text {II }}$ as region III, which evolves exactly as the EdS universe. For the uncompensated model, we set the initial coordinate density in region III to the initial EdS background density $\rho_{\text {EdS }, i}$.

Because the geodesic equation involves $R^{\prime \prime}$, a discontinuous $R^{\prime \prime}$ (such as those conforming with top-hat density profiles) will deflect photons in coordinate space (but not in physical space) as they travel across the discontinuity. This does not have theoretical impact on the investigation, but to ensure the numerical precision, one must accurately determine the 4 -coordinate $x^{\mu}$ where the photon crosses the discontinuity and then adjust its wavevector $k^{\mu}$, so that its physical velocity remains the same and $k^{\mu} k_{\mu}=0$ across the discontinuity. We avoid such a numerical issue by designing a curvature function that is continuous to at least second order (so are $R$ and $M$ ), which renders smooth density profiles via Equation (4). The functional forms of $K$ and corresponding density profiles are given in Appendix A. In summary, the parameters of the toy models are $r_{\mathrm{I}}, r_{\mathrm{II}}$, and the initial overdensity of region I, $\delta_{\mathrm{I}, i} \equiv \rho_{\mathrm{I}, i} / \rho_{\mathrm{EdS}, i}-1$.

Although there is no peculiarity analytically as the curvature function $K$ approaches 0 , e.g., just inside of $r_{\text {II }}$ in the compensated models, Equations (5) and (6) are not suitable for numerical evaluation when $|K|$ is very small. Observing that $q \equiv u|K|^{-1 / 2}$ remains finite as $|K| \rightarrow 0$, we Taylor expand Equations (5) and (6) around $u=0$ and then replace $u$ with $q|K|^{1 / 2}$ when $K \sim 0$.

\section{GEODESICS}

The geodesic equation is given by

$$
\frac{d^{2} x^{\mu}}{d v^{2}}+\Gamma_{\alpha \beta}^{\mu} \frac{d x^{\alpha}}{d v} \frac{d x^{\beta}}{d v}=0,
$$

where $x^{\mu}=(t, r, \theta, \phi), v$ is the affine parameter, and $\Gamma_{\alpha \beta}^{\mu}$ is the Christoffel symbol. In the LTB metric, Equation (9) becomes

$$
\begin{aligned}
\frac{d k^{t}}{d v}= & -\frac{R^{\prime} \dot{R}^{\prime}}{1-K r^{2}}\left(k^{r}\right)^{2}-R \dot{R}\left(k^{\Omega}\right)^{2} \\
\frac{d k^{r}}{d v}= & -\frac{2 \dot{R}^{\prime}}{R^{\prime}} k^{t} k^{r}-\left[\frac{R^{\prime \prime}}{R^{\prime}}+\frac{2 K r+K^{\prime} r^{2}}{2\left(1-K r^{2}\right)}\right]\left(k^{r}\right)^{2} \\
& +R \frac{1-K r^{2}}{R^{\prime}}\left(k^{\Omega}\right)^{2} \\
\frac{d k^{\theta}}{d v}= & -\frac{2}{R} \frac{d R}{d v} k^{\theta}+\sin \theta \cos \theta\left(k^{\phi}\right)^{2} \\
\frac{d k^{\phi}}{d v}= & -\frac{2}{R} \frac{d R}{d v} k^{\phi}-2 \cot \theta k^{\theta} k^{\phi}
\end{aligned}
$$

where $k^{\mu} \equiv d x^{\mu} / d v$ is the wavevector, $\left(k^{\Omega}\right)^{2} \equiv\left(k^{\theta}\right)^{2}+$ $\sin ^{2} \theta\left(k^{\phi}\right)^{2}$, and $d R / d v=\dot{R} k^{t}+R^{\prime} k^{r}$. We enumerate the index $\mu$ explicitly as $t, r, \theta$, and $\phi$ instead of numbers for clarity. Because of spherical symmetry, we only need to study geodesics in the equatorial plane.

The frequency of a photon with wavevector $k^{\mu}$ as measured by an observer (or source) with 4 -velocity $U^{\mu}$ is

$$
\nu \propto-k^{\mu} U_{\mu} \text {. }
$$


If both the observer and the source are comoving in the LTB metric, then the photon redshift is given by

$$
1+z=\frac{k^{t}\left(t_{\mathrm{e}}, r_{\mathrm{e}}\right)}{k^{t}\left(t_{\mathrm{o}}, r_{\mathrm{o}}\right)},
$$

where the subscripts e and o denote the event of emission and that of observation, respectively, and $k_{\mathrm{e}}^{t} \equiv$ $k^{t}\left(t_{\mathrm{e}}, r_{\mathrm{e}}\right)=1$ is set arbitrarily as the initial condition. In actual calculations, we propagate photons backward from the observer to the source surface by setting $v \rightarrow-v$ (hence $k^{\mu} \rightarrow-k^{\mu}$ ) and the initial condition $k_{\mathrm{o}}^{t} \equiv k^{t}\left(t_{\mathrm{o}}, r_{\mathrm{o}}\right)=-1$. While Equations (9-15) remain the same under the reversal of $v$, others may change sign. To avoid confusion, we only refer to the forward case in all the equations and discussions below.

The RS effect under investigation is of the order $|\Delta T| / T=|\Delta z| /(1+z) \sim 10^{-6}-10^{-5}$, so one must ensure that numerical errors in $k^{t}$ is much less than one part in a million. Numerical results can be easily checked in uniform regions where alternative solutions exist. In addition, we perform several general tests that may detect numerical errors, which are described as follows.

By definition, null geodesics obey $k_{\mu} k^{\mu}=0$. This is a redundant constraint once the initial condition is set, as in principle the geodesic equation does not induce violation of the condition. However, numerical errors could be accumulated. While enforcing redundant constraints numerically is a subject of research itself, we simply adjust the time steps so that $\left|k_{\mu} k^{\mu} / k_{t} k^{t}\right| \lesssim 10^{-8}$ when the photons exit the systems. This condition is not sufficient to validate the results, but violation of it indicates significant numerical errors.

In spherically symmetric systems, each geodesic remains in a plane. One can always rotate the coordinates to place the geodesic in the equatorial plane, in which case $R^{2} k^{\phi}$ is conserved. Similarly, $R^{2} k^{\theta}$ is conserved if $k^{\phi}=0$. We do not apply the known solution to reduce the dimensions of the system. Rather, we use them to check the precision of the numerical solutions. The maximum fractional error of the conserved quantity along off-center geodesics is found to be $\sim 10^{-9}$.

Finally, radial geodesics can be calculated directly from the LTB metric Equation (1)

$$
\frac{d t}{d r}= \pm \sqrt{\frac{R^{\prime 2}}{1-K r^{2}}},
$$

where outward (inward) geodesics take the positive (negative) sign. By differentiating Equation (15) with respect to $r(v)$, one gets the evolution of redshift

$$
\begin{aligned}
\frac{d \ln (1+z)}{d r} & =-\frac{1}{k_{\mathrm{o}}^{t}} \frac{d k_{\mathrm{o}}^{t}}{d r}=-\left.\frac{1}{k_{\mathrm{o}}^{t}} \frac{d v}{d r}\right|_{\mathrm{o}} \frac{d k_{\mathrm{o}}^{t}}{d v} \\
& =\frac{1}{k_{\mathrm{o}}^{t} k_{\mathrm{o}}^{r}} \Gamma_{\alpha \beta}^{t} k_{\mathrm{o}}^{\alpha} k_{\mathrm{o}}^{\beta}= \pm \frac{\dot{R}^{\prime}}{\sqrt{1-K r^{2}}}
\end{aligned}
$$

where the sign on the far right side is the same as that in Equation (16) (see Mustapha et al. 1998; Célérier 2000, for an alternative derivation). Fractional differences between numerical solutions of $z(r)$ and $t(r)$ using Equations (10-11) and those using Equations (16) and (17) are $\lesssim 10^{-8}$ when photons exit the model structures.

\section{REES-SCIAMA EFFECT OF SUPER STRUCTURES}

The clusters studied in Granett et al. 2008 have radii of roughly $100 h^{-1} \mathrm{Mpc}$ and a mean galaxy overdensity of 0.72 within the overdense region, corresponding to a matter overdensity of 0.36 with a galaxy clustering bias of roughly 2 for luminous red galaxies (Padmanabhan et al. 2007; Blake et al. 2008). Thus, our simple LTB models of these clusters have an inner radius $r_{\mathrm{I}}=140 \mathrm{Mpc}^{1}$, an outer radius $r_{\mathrm{II}}=280 \mathrm{Mpc}(205 \mathrm{Mpc})$ for the compensated (uncompensated) profile, and a uniform overdensity $\delta_{\mathrm{I}}=0.36$ in region I at $z=0.5$. For voids, the only difference is $\delta_{\mathrm{I}}=-0.19$. Appendix A provides more details about these profiles and their corresponding metric quantity $R$.

To calculate the RS effect, we place a model structure between an observer and a source surface and propagate photons from the latter to the former (as mentioned in Section 3, they are actually propagated backwards). The source surface is spherical around the observer in coordinate space, so that in absence of the structure, photons originated from the surface at the same time would reach the observer at the same time with the same redshift. With the intervening structure though, the photons received at the same time would have started from the source surface at different times because of gravitational time delay, and their redshifts would have to compensate for the time delay plus additional changes because of nonlinear evolution of the structure. The net change in temperature is a sum of the two components:

$$
\Delta T=-T_{\mathrm{CMB}}\left[H_{\mathrm{s}} \Delta t_{\mathrm{s}}+\frac{\Delta z_{\mathrm{s}}}{1+z_{\mathrm{s}}}\right],
$$

where $T_{\mathrm{CMB}}=2.73 \mathrm{~K}$ is the present $\mathrm{CMB}$ temperature, $\Delta t_{\mathrm{s}}$ is the time difference at the source, $H_{\mathrm{s}} \equiv H\left(z_{\mathrm{s}}\right)$ is the Hubble parameter at the source redshift $z_{\mathrm{s}}$, and $\Delta z_{\mathrm{s}}$ is the redshift difference "measured" by an observer at present. Note that the two components in Equation (18) cannot be measured separately in the case of the CMB. The time component accounts for the decrease of the CMB temperature with time, and $\Delta z_{\mathrm{s}}$ bears the subscript $\mathrm{s}$ because it varies with the source location.

In the uniform background universe, a small time difference of $\Delta t$ translates to a redshift difference of

$$
\Delta z=-(1+z) H(z) \Delta t
$$

Hence, in region III of compensated models, one can separate $z_{\mathrm{s}}$ into a time-compensation term

$$
z_{\mathrm{s}}^{t}=-\left(1+z_{\mathrm{s}}\right) H_{\mathrm{s}} \Delta t_{\mathrm{s}}
$$

and an evolution term $z_{\mathrm{s}}^{e}=\Delta z_{\mathrm{s}}-z_{\mathrm{s}}^{t}$. Since $z_{\mathrm{s}}^{t}$ exactly cancels $H_{\mathrm{s}} \Delta t_{\mathrm{s}}$ in region III, Equation (18) becomes

$$
\Delta T=-T_{\mathrm{CMB}} \frac{\Delta z_{\mathrm{s}}^{e}}{1+z_{\mathrm{s}}} .
$$

In the compensated models, the only place where $\mathrm{CMB}$ photons are affected by the structure is within $r_{\mathrm{II}}$. Hence, the RS effect (and ISW effect in general) does not depend on the location of the hypothetical source surface as long as the compensated structure is enclosed between the observer and the source. In other words, $z_{\mathrm{s}}^{e}$ scales linearly

${ }^{1}$ We adopt $h=0.71$ here only to obtain the size of the observed structures in units of Mpc. 


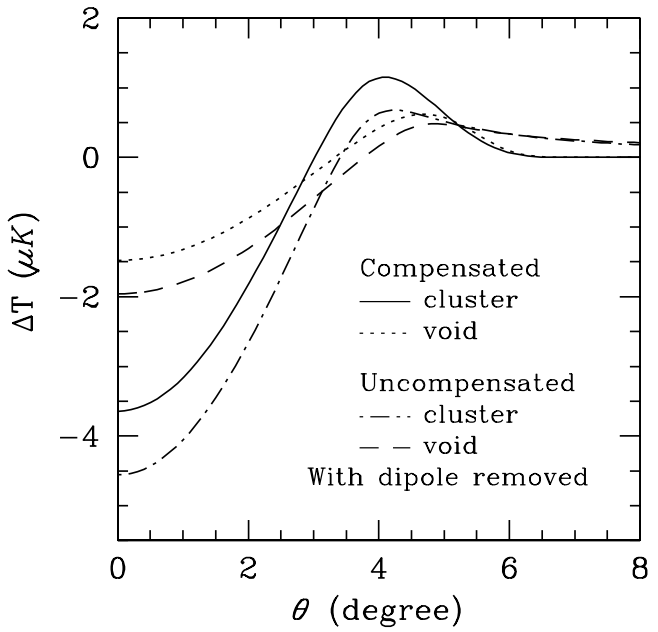

FIG. 1.- CMB temperature shifts due to intervening model structures at $z=0.5$, which have similar sizes $\left(r_{\mathrm{I}}=140 \mathrm{Mpc}\right.$ or $\left.\sim 4^{\circ}\right)$ and overdensities $\left(\delta_{\mathrm{I}}=0.36,-0.19\right)$ as the clusters and voids in Granett et al. (2008). The average densities of the compensated cluster (solid line) and void (dotted line) within the outer radius $r_{\mathrm{II}}=280 \mathrm{Mpc}$ equal the background density. The uncompensated cluster (dot-dashed line) and void (dashed line) have very similar inner profiles $\left(r<1.4 r_{\mathrm{I}}\right)$ as their compensated counterparts (see Figure 4). The most prominent feature of the uncompensated models is a CMB dipole, which has been removed.

with $1+z_{\mathrm{s}}$ in the uniform background. For the uncompensated models, the density evolution just beyond $r_{\mathrm{II}}$ is still significantly different from that in the EdS universe, so we place the source far away from the model structure.

Figure 1 illustrates the RS effect of four LTB model structures. The main feature is a temperature decrement of several $\mu \mathrm{K}$ in the inner region and a slight temperature increase at the outskirt, regardless whether the structure is a cluster or a void. This is distinct from the linear ISW effect in a universe dominated by dark energy, which generally causes a temperature increase for clusters and a decrement for voids. The RS effect of the voids may seem counterintuitive at first, and we find that it is the result of underdensities generally evolving slower than the expansion rate in the EdS universe.

Because a distant comoving observer or emitter in the uncompensated LTB models would be seen to have a peculiar velocity in the EdS background, the most prominent feature of these models is a CMB temperature dipole. Once the dipole is subtracted, the temperature profiles are similar to those of the compensated models.

As mentioned in Section 2, we set a low value for the Hubble constant in the EdS background. If we used instead $H_{0}=71 \mathrm{~km} \mathrm{~s}^{-1} \mathrm{Mpc}^{-1}$, the central temperature decrement of the compensated cluster (void) in Figure 1 would become $9.9 \mu \mathrm{K}(4.2 \mu \mathrm{K})$, nearly tripling the decrement of $3.6 \mu \mathrm{K}(1.5 \mu \mathrm{K})$ with $H_{0}=50 \mathrm{~km} \mathrm{~s}^{-1} \mathrm{Mpc}^{-1}$.

Figure 2 shows the dependence of the RS effect on the model parameters $r_{\mathrm{I}}, r_{\mathrm{II}}$, and $\delta_{\mathrm{I}}$. We use the difference between the characteristic peak and trough of the temperature profile $\left(T_{+}-T_{-}\right)$in Figure 1 to represent the amplitude of the RS effect and show results for compensated clusters. Because it takes more time for CMB photons to go through a larger structure, its potential can evolve more to produce stronger RS effects. Structures with larger $\left|\delta_{\mathrm{I}}\right|$ evolve faster and also produce larger RS effects. These are indeed seen in Figure 2. Moreover, the

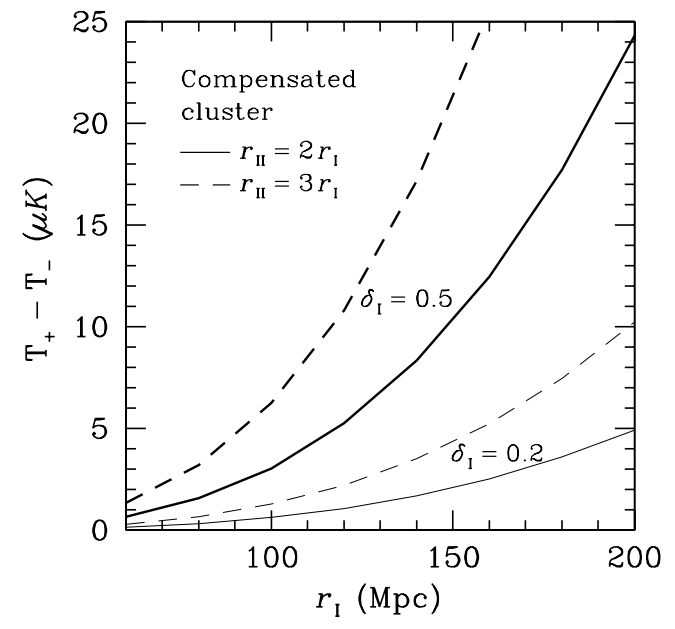

FIG. 2.- Amplitude of the RS effect of compensated clusters as a function of the inner radius with $r_{\mathrm{II}}=2 r_{\mathrm{I}}$ (solid lines) and $r_{\mathrm{II}}=3 r_{\mathrm{I}}$ (dashed lines). Thick lines are for clusters with $\delta_{\mathrm{I}}=0.5$ at $z=0.5$, while thin lines are for $\delta_{\mathrm{I}}=0.2$.

RS effect increases fairly rapidly with $r_{\mathrm{I}}, r_{\mathrm{II}}$, and $\delta_{\mathrm{I}}$.

The several $\mu \mathrm{K}$ level RS effect in Figure 1 is significant compared to the detected CMB temperature shift of $\sim$ $\pm 10 \mu \mathrm{K}$ due to super structures and is comparable to the expected maximum linear ISW effect of $4.2 \mu \mathrm{K}$ within an aperture of $100 h^{-1} \mathrm{Mpc}$ in the Millennium simulation (Granett et al. 2008). Although the RS effect could bring the expected total ISW effect of the supervoids more in line with the observed value, it would also widen the discrepancy between the observation and the expected total ISW effect for superclusters.

\section{EFFECT OF EVOLVING STRUCTURES ON GALAXY REDSHIFTS}

Figure 3 illustrates the time delay component (dotted lines) and the redshift component (dashed line) of the RS effect (circles) as given in Equation (18). These two effects are opposite to each other and are of the same order, so that the net result is much smaller than either one. In other words, the gravitational time delay in the potential is a more dominant effect than the potential evolution for super structures.

Treating recombination as an instantaneous event, which suffices our purpose, one can view the RS effect and the ISW effect in general as a single redshift effect without time delay. This view is fully equivalent to that of Rees \& Sciama (1968). However, one is no longer comparing photons that have started from the same spatial coordinates at different times; rather, the CMB photons received at the same time were emitted at the same instance of recombination, but they must have started from different locations. In other words, the last scattering surface as viewed by the observer is no longer spherical in presence of inhomogeneities.

To cross-check the results, we trace photon geodesics to the same time surface rather than the same spatial coordinate surface described in Section 4. This is analogous to theoretical calculations of the ISW effect by integrating over (conformal) time. The resulting change in the temperature is then given solely by the redshift difference, i.e., a change in $k^{t}$. As shown in Figure 3, this single redshift effect (solid lines) gives exactly the same RS effect as that from the combination of the time delay 


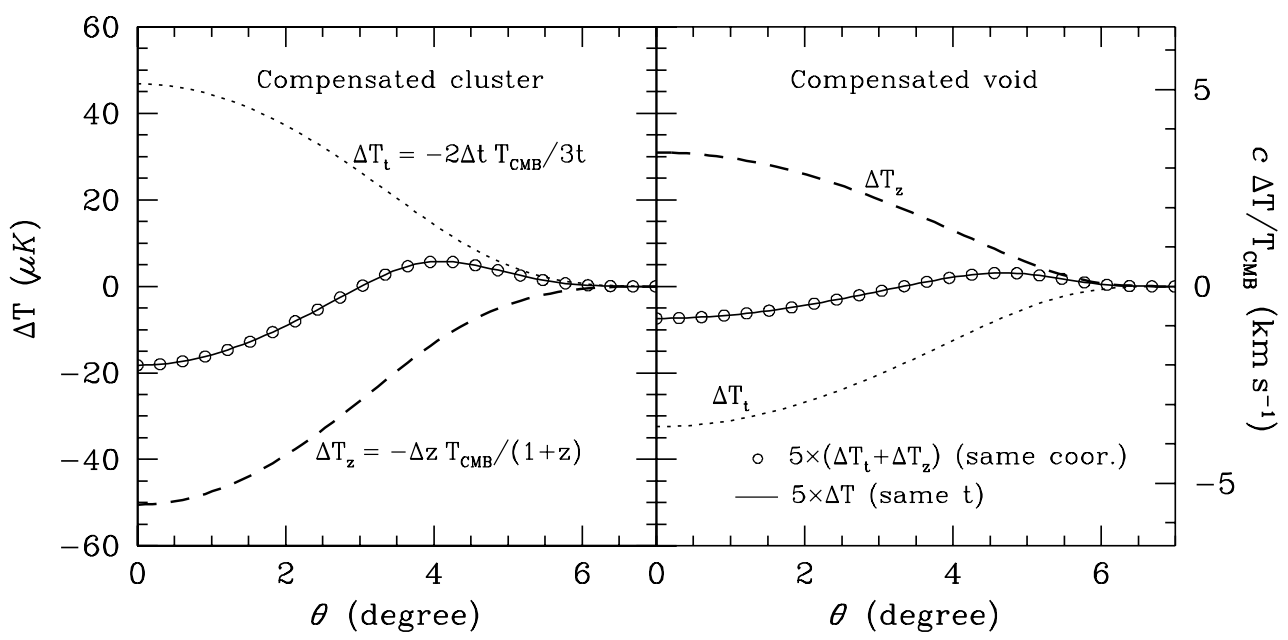

FIG. 3. - Two equivalent views of the RS effect: a combination (circles) of the redshift (dashed lines, $\Delta T_{z}$ ) and time delay (dotted line, $\Delta T_{t}$ ) effects on photons started at the same coordinate distance from the observer and a single redshift effect of photons started out at the same time (solid lines), e.g., the instance of recombination. The left panel and the right panel are for the compensated cluster and the compensated void, respectively. The combined RS results (circles) are the same as those in Figure 1 but amplified by 5 times for better viewing. For galaxies, their redshift changes are given by $(1+z) \Delta T_{z} / T_{\mathrm{CMB}}$, i.e., slightly more redshifted behind clusters and less behind voids in the EdS universe.

and redshift effects (circles).

Unlike CMB photons, light from a comoving galaxy must be emitted from the same coordinates whether there is an intervening structure or not. Furthermore, galaxy redshifts can be directly measured through spectroscopy, so that the redshift effect is no longer mixed with the time delay effect. Without the large cancellation from time delay, changes of galaxy redshifts can be roughly an order of magnitude greater than fractional changes in the CMB temperature. For the clusters (voids) studied in Section 4, the redshift (blueshift) effect is merely several kilometers per second, or at the percent level of the large-scale rms bulk velocity, estimated to be a few hundred kilometers per second (e.g., Zhan \& Fang 2002). Nevertheless, it might be detectable statistically in the future and could be somewhat significant to largescale redshift surveys that aim to precisely measure the redshift distortion effect.

\section{DISCUSSION AND CONCLUSIONS}

We have studied the intrinsic RS effect of super structures due to their weakly nonlinear evolution using idealized LTB models and find that clusters and voids resembling those in Granett et al. (2008) can cause CMB temperature decrements of a few $\mu K$ in the EdS universe. The amplitude of the RS effect is fairly significant compared to the measured CMB temperature changes, so one needs to take into account both the RS effect and the linear ISW effect when interpreting the observations.

It is interesting to note that the RS effect is a central CMB temperature decrement, regardless whether the structure is a cluster or a void. The reason is that generally overdensities grow faster than the cosmic expansion in the EdS universe, while underdensities evolves slower than the cosmic expansion.

In the concordant $\Lambda$ CDM universe, the linear ISW effect is expected to be stronger than the RS effect at low redshift when dark energy is dominant. A full calculation of the total ISW effect with Equation (9) is rather involved, as the metric function $R$ and its first and second derivatives are no longer analytic in the $\Lambda$ CDM universe.
We can estimate the very effect along radial geodesics using Equations (16) and (17). The required $\dot{R}$ and $\dot{R}^{\prime}$ evolve according to

$$
\begin{aligned}
\ddot{A} & =H_{0}^{2}\left(1-\Omega_{\mathrm{m}}\right) A-\frac{H_{0}^{2} \Omega_{\mathrm{m}}+a_{i} K}{2 A^{2}} \\
\dot{A}^{\prime} & =\frac{\ddot{A} A^{\prime}}{\dot{A}}+\frac{K^{\prime}}{2 \dot{A}}\left(\frac{a_{i}}{A}-1\right),
\end{aligned}
$$

where $A=r^{-1} R$, and $\Omega_{\mathrm{m}}=0.3$ is the present matter fraction of the background flat $\Lambda$ CDM universe. The initial conditions are given by Equation (8), and the Hubble constant is set to $70 \mathrm{~km} \mathrm{~s}^{-1} \mathrm{Mpc}^{-1}$ in this case. For the compensated cluster (void) in Figure 3, we find that $\Delta T_{z}=-21.5 \mu \mathrm{K}(13.3 \mu \mathrm{K}), \Delta T_{t}=26.6 \mu \mathrm{K}(-18.7 \mu \mathrm{K})$, and the net temperature shift $\Delta T=5.1 \mu \mathrm{K}(-5.4 \mu \mathrm{K})$ through its center. These results agree with the general expectation that photons gain (lose) energy going through a decaying potential well (wall) in the accelerating $\Lambda$ CDM universe.

For galaxies, the redshift effect is separable from the time delay effect, and the magnitude of the change in redshift is much larger than the fractional change in the CMB temperature. This redshift effect is due in most part to the compensation for the time delay effect, e.g., a galaxy seen at an earlier time also has a higher redshift. Evolution of the potential plays a minor role in the case of super structures. Hence, the effect on the galaxy redshift is different from the RS effect or the ISW effect.

The compensated cluster (void) in Figure 3 changes the redshift of a $z_{\mathrm{s}} \simeq 0.6$ background galaxy by $\Delta z_{\mathrm{s}}=$ $2.8 \times 10^{-5}\left(-1.7 \times 10^{-5}\right)$ in the EdS universe and by $\Delta z_{\mathrm{s}}=1.2 \times 10^{-5}\left(-7.5 \times 10^{-6}\right)$ in the $\Lambda$ CDM universe. Although measuring such redshift differences is not completely out of technical reach, the fact that there is not a standard redshift to compare with makes it impossible to measure this effect on individual galaxies. Since it is a systematic perturbation to galaxy redshifts, one might be able to detect the effect by comparing the galaxy redshift distribution with and without intervening struc- 
tures. However, many issues come into play. For example, magnification by the very foreground structures also affects how background galaxies are selected into the sample and thus change the galaxy redshift distribution perhaps more significantly.

While the absolute time delay due to foreground structures is not directly observable, one can obtain the relative time delay between images of a strongly lensed source either by actual measurements with a variable source or by estimation with a lens model. This could have allowed a short cut to the Sandage-Loeb test that has to run for decades. Assuming for the moment that the potential does not evolve, i.e., $\Delta T=0$ in Equation (18), we have

$$
\Delta v=\frac{c \Delta z_{\mathrm{s}}}{1+z_{\mathrm{s}}}=-c H_{\mathrm{s}} \Delta t_{\mathrm{s}}=-\frac{c \Delta t_{0}}{1+z_{\mathrm{s}}} H_{\mathrm{s}}
$$

where $\Delta t_{0}$ is the relative time delay observed at $z=0$. For a source at $z_{\mathrm{s}}=1$, the velocity difference corresponding to a time delay of one year is $-1.8 \mathrm{~cm} \mathrm{~s}^{-1}$. The evolution of the lens potential also contributes to the redshift difference. Because lenses are more or less virialized structures, one might approximate them as static objects in physical space and determine their potential evolution in the expanding universe. One might also stack CMB measurements behind a large number of similar lenses to determine the potential evolution.

For comparison, the Sandage-Loeb test gives (Loeb 1998)

$$
\Delta v=-\frac{c \Delta t_{0}}{1+z_{\mathrm{s}}}\left[H_{\mathrm{s}}-H_{0}(1+z)\right] .
$$

Here $\Delta t_{0}$ is the time between two epochs of measurements. In the $\Lambda$ CDM universe, $H_{\mathrm{s}} \lesssim H_{0}(1+z)$ at $z_{\mathrm{s}} \lesssim 2.5$, so the direction of the redshift change in the strong-lensing case is opposite to the Sandage-Loeb test at $z_{\mathrm{s}} \lesssim 2.5$. The amplitude in the strong-lensing case is almost an order of magnitude larger than that in the Sandage-Loeb test, making the former comparatively easier to measure for the same $\Delta t_{0}$. Moreover, the Sandage-Loeb test requires extremely high-precision absolute calibration of the instruments over a few decades, which is more difficult than high-precision relative measurements performed at the same time using the same instrument in the strong lensing case.

Unfortunately, as Loeb (1998) pointed out, differences in the conditions of the images (e.g., shape, magnification, noise, etc.) of the same galaxy could already cause larger differences in the redshift measurements; even more problematically, proper motion of the lens could induce a difference in image redshifts (Birkinshaw \& Gull 1983) far exceeding that associated with the time delay. Therefore, even though the strong lensing case has a few advantages, it is still impractical to use the redshift difference and time delay between images of a strongly lensed source to measure the Hubble expansion at the source redshift.

This work was supported by the Bairen program from the Chinese Academy of Sciences, the National Basic Research Program of China grant No. 2010CB833000, and the National Natural Science Foundation of China grant No. 11033005.

\section{APPENDIX}

\section{MODEL STRUCTURES}

We require the curvature function $K$ of the model structures to have a continuous second derivative with respect to $r$. For compensated models, we specify $K$ directly

$$
K= \begin{cases}K_{\mathrm{I}} & 0 \leq r<r_{\mathrm{I}} \\ K_{\mathrm{I}}\left(\frac{r_{\mathrm{II}}-r}{r_{\mathrm{II}}-r_{\mathrm{I}}}\right)^{3}\left[4+3 \frac{\left(r-2 r_{\mathrm{I}}+r_{\mathrm{II}}\right)\left(2 r-r_{\mathrm{I}}-r_{\mathrm{II}}\right)}{\left(r_{\mathrm{II}}-r_{\mathrm{I}}\right)^{2}}\right] & r_{\mathrm{I}} \leq r<r_{\mathrm{II}} \\ 0 & r \geq r_{\mathrm{II}}\end{cases}
$$

where $K_{\mathrm{I}}=\delta_{\mathrm{I}, i} a_{i}^{2} H_{i}^{2} \Omega_{\mathrm{m}}$, and $\delta_{\mathrm{I}, i}$ is the initial overdensity of region I. For uncompensated models, we let the initial overdensity $\delta_{i}$ follow the same form as $K$ (with $K_{\mathrm{I}}$ replaced by $\delta_{\mathrm{I}, i}$ ) and then use Equations (4), (8), and (2) to obtain $K$. We choose $r_{\text {II }}$ for uncompensated models so that the uncompensated overdensity profiles roughly match those of compensated models in the central region.

The left panel of Figure 4 shows overdensity profiles of the compensated (dotted lines) and uncompensated (solid lines) cluster models. For both models, the initial overdensity of region I at $a_{i}$ is set to $\delta_{\mathrm{I}, i}=7.0 \times 10^{-4}$, so that $\delta_{\mathrm{I}}=0.36$ at redshift $z=0.5$ to match the mean overdensity of overdense regions of the clusters found in Granett et al. (2008). The density of the uncompensated cluster grows with time everywhere. The void models behave similarly (not shown) but with inverted profiles and smaller amplitudes. Moreover, the uncompensated void develops slightly positive overdensity $\left(\delta \ll\left|\delta_{\mathrm{I}}\right|\right)$ at $r \gtrsim r_{\text {II }}$ at late time.

The right panel of Figure 4 illustrates the evolution of the function $R / a r$ for the compensated cluster (solid lines) and the compensated void (dotted lines). The void has $\delta_{\mathrm{I}, i}=-5.5 \times 10^{-4}$ and $\delta_{\mathrm{I}}=-0.19$ at $z=0.5$, matching the mean overdensity of underdense regions of the voids in Granett et al. (2008). In our setup, underdense regions always expand faster than the background after $t_{i}$. Hence, for the voids, $R(t, r) \geq a r$ at $t>t_{i}$, and the equality takes place where the mean overdensity within $r$ vanishes. Similarly, for the clusters, $R(t, r) \leq a r$ at $t>t_{i}$.

\section{REFERENCES}

Afshordi, N., Loh, Y.-S., \& Strauss, M. A. 2004, Phys. Rev. D, 69,083524

Afshordi, N., Slosar, A., \& Wang, Y. 2011, Journal of Cosmology and Astro-Particle Physics, 1, 19
Alnes, H., \& Amarzguioui, M. 2006, Phys. Rev. D, 74, 103520

Alnes, H., Amarzguioui, M., \& Grøn, Ø. 2006, Phys. Rev. D, 73, 083519

Bennett, C. L. et al. 2003, ApJS, 148, 97 

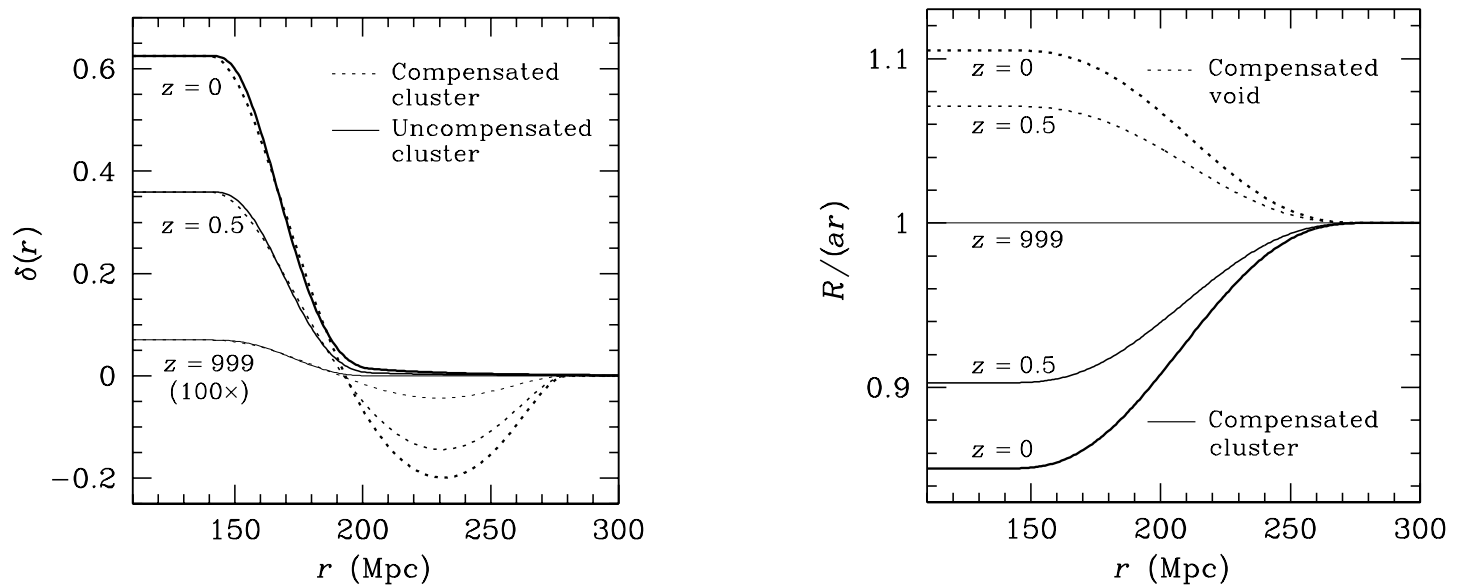

FIG. 4. - Left panel: Overdensity profiles of the compensated (dotted lines) and uncompensated (solid lines) cluster models. The compensated model has $r_{\mathrm{I}}=140 \mathrm{Mpc}$ and $r_{\mathrm{II}}=280 \mathrm{Mpc}$, while the uncompensated model has $r_{\mathrm{I}}=140 \mathrm{Mpc}$ and $r_{\mathrm{II}}=205 \mathrm{Mpc}$. Profiles of the voids are similar to their corresponding cluster profiles but are inverted with lower amplitudes. Right panel: Evolution of the function $R / a r$ of the compensated cluster (solid lines) and the compensated void (dotted lines). The initial condition at $a_{i}=10^{-3}$ sets $R_{i}=a_{i} r$. The uncompensated models behave similarly as their compensated counterpart but converge to $R / a r=1$ only at $r=\infty$.

Birkinshaw, M., \& Gull, S. F. 1983, Nature, 302, 315

Birkinshaw, M., Hughes, J. P., \& Arnaud, K. A. 1991, ApJ, 379, 466

Blake, C., Collister, A., \& Lahav, O. 2008, MNRAS, 385, 1257

Bondi, H. 1947, MNRAS, 107, 410

Boughn, S., \& Crittenden, R. 2004, Nature, 427, 45

Cai, Y.-C., Cole, S., Jenkins, A., \& Frenk, C. 2009, MNRAS, 648

Cai, Y.-C., Cole, S., Jenkins, A., \& Frenk, C. S. 2010, MNRAS, 407, 201

Carlstrom, J. E., Joy, M., \& Grego, L. 1996, ApJ, 456, L75+

Célérier, M.-N. 2000, A\&A, 353, 63

Fosalba, P., Gaztañaga, E., \& Castander, F. J. 2003, ApJ, 597, L89

Giannantonio, T., Scranton, R., Crittenden, R. G., Nichol, R. C., Boughn, S. P., Myers, A. D., \& Richards, G. T. 2008,

Phys. Rev. D, 77, 123520

Granett, B. R., Neyrinck, M. C., \& Szapudi, I. 2008, ApJ, 683, L99

—. 2009, ApJ, 701, 414

Gurvits, L. I., \& Mitrofanov, I. G. 1986, Nature, 324, 349

Inoue, K. T., \& Silk, J. 2006, ApJ, 648, 23

Kolb, E. W., Marra, V., \& Matarrese, S. 2008, Phys. Rev. D, 78, 103002

Lemaître, G. 1933, Annales de la Societe Scietifique de Bruxelles, 53,51

Loeb, A. 1998, ApJ, 499, L111+

Marra, V., Kolb, E. W., Matarrese, S., \& Riotto, A. 2007, Phys. Rev. D, 76, 123004

Martinez-Gonzalez, E., \& Sanz, J. L. 1990, MNRAS, 247, 473

Maturi, M., Dolag, K., Waelkens, A., Springel, V., \& Enßlin, T. 2007, A\&A, 476, 83
Mustapha, N., Bassett, B. A. C. C., Hellaby, C., \& Ellis, G. F. R. 1998, Classical and Quantum Gravity, 15, 2363

Padmanabhan, N., Hirata, C. M., Seljak, U., Schlegel, D. J., Brinkmann, J., \& Schneider, D. P. 2005, Phys. Rev. D, 72, 043525

Padmanabhan, N. et al. 2007, MNRAS, 378, 852

Panek, M. 1992, ApJ, 388, 225

Paranjape, A., \& Singh, T. P. 2008, Journal of Cosmology and Astro-Particle Physics, 3, 23

Rees, M. J., \& Sciama, D. W. 1968, Nature, 217, 511

Sachs, R. K., \& Wolfe, A. M. 1967, ApJ, 147, 73

Sakai, N., \& Inoue, K. T. 2008, Phys. Rev. D, 78, 063510

Sandage, A. 1962, ApJ, 136, 319

Seljak, U. 1996, ApJ, 460, 549

Smith, R. E., Hernandez-Monteagudo, C., \& Seljak, U. 2009, ArXiv e-prints, 0905.2408

Spergel, D. N. et al. 2003, ApJS, 148, 175

Stebbins, A. 1988, ApJ, 327, 584

Sunyaev, R. A., \& Zeldovich, Y. B. 1972, Comments on Astrophysics and Space Physics, 4, 173

Thompson, K. L., \& Vishniac, E. T. 1987, ApJ, 313, 517

Tolman, R. C. 1934, Proceedings of the National Academy of Science, 20, 169

Tomita, K., \& Inoue, K. T. 2008, Phys. Rev. D, 77, 103522

Tuluie, R., Laguna, P., \& Anninos, P. 1996, ApJ, 463, 15

Valkenburg, W. 2009, Journal of Cosmology and Astro-Particle Physics, 6, 10

Zhan, H., \& Fang, L. 2002, ApJ, 566, 9 